\title{
Texture analysis of orbital magnetic resonance imaging for monitoring and predicting treatment response to glucocorticoids in patients with thyroid-associated ophthalmopathy
}

\author{
Yue-Yue Wang ${ }^{1, *}$, Qian Wu ${ }^{2, *}$, Lu Chen ${ }^{2, *}$, Wen Chen², Tao Yang${ }^{1}$, Xiao-Quan Xu², Fei-Yun $\mathrm{Wu}^{2}$, Hao Hu${ }^{2}$ and \\ Huan-Huan Chen (101
}

'Department of Endocrinology, The First Affiliated Hospital of Nanjing Medical University, Nanjing, China

2Department of Radiology, The First Affiliated Hospital of Nanjing Medical University, Nanjing, China

Correspondence should be addressed to H Hu or H-H Chen: haohu_njmu@foxmail.com or drchenhuanhuan@njmu.edu.cn

\begin{abstract}
Purpose: To evaluate the value of MRI-based texture analysis of extraocular muscle (EOM) and orbital fat (OF) in monitoring and predicting the response to glucocorticoid (GC) therapy in patients with thyroid-associated ophthalmopathy (TAO).

Methods: Thirty-seven active and moderate-to-severe TAO patients (responders, $n=23$; unresponders, $n=14$ ) were retrospectively enrolled. MRI-based texture parameters (entropy, uniformity, skewness and kurtosis) of EOM and OF were measured before and after GC therapy, and compared between groups. Correlations between the changes of clinical activity score (CAS) and imaging parameters before and after treatment were assessed. Receiver operating characteristic curves were used to evaluate the predictive value of identified variables.

Results: Responsive TAOs showed significantly decreased entropy and increased uniformity at EOM and OF after GC therapy $(P<0.01)$, while unresponders showed no significance. Changes of entropy and uniformity at EOM and OF were significantly correlated with changes of CAS before and after treatment $(P<0.05)$. Responders showed significantly lower entropy and higher uniformity at EOM than unresponders before treatment $(P<0.01)$. Entropy and uniformity of EOM and disease duration were identified as independent predictors for responsive TAOs. Combination of all three variables demonstrated optimal efficiency (area under curve, 0.802 ) and sensitivity (82.6\%), and disease duration alone demonstrated optimal specificity (100\%) for predicting responsive TAOs.

Conclusion: MRI-based texture analysis can reflect histopathological heterogeneity of orbital tissues. It could be useful for monitoring and predicting the response to GC in TAO patients.
\end{abstract}

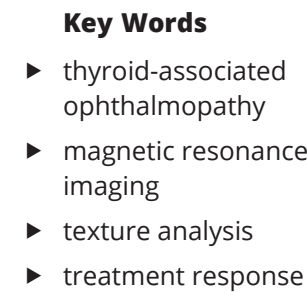

Endocrine Connections (2021) 10, 676-684

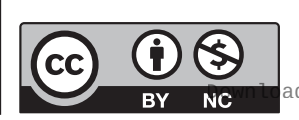

This work is licensed under a Creative Commons Attribution-NonCommercial 4.0 International License. ded from Bioscientifica.com at 04/26/2023 01:07:56PM 


\section{Introduction}

Thyroid-associated ophthalmopathy (TAO) is an autoimmune and inflammatory orbital disease (1). The natural course of TAO is composed of an initial active stage and a later static stage. Systemic immunosuppressive treatment is the first-line administration in the active stage, and intravenous glucocorticoids (GC) have been the mainstay of treatment for moderate-to-severe and active cases (2). However, the response rate of intravenous GC was reported as only $70-80 \%(3,4)$. Quite a few patients would not achieve significant improvements even after strict screening and standard treatment. Unresponsive patients would suffer from a series of untoward effects, and even deterioration of the disease (5). Therefore, determining a way to monitor and predict the treatment response to GC is clinically desired, in order to select individuals who need close follow-up and replacement therapy.

Currently, clinical activity score (CAS) is widely used to grade the activity of TAO and guide treatment decisions (6), but the subjective evaluation sometimes limits its efficacy. Moreover, the assessment of CAS is confined to the anterior orbit, which may underestimate disease pathogenesis at the posterior orbit. Due to the ability of objectively and overall revealing changes within the whole orbit, MRI has been increasingly used in the assessment of $\operatorname{TAO}(7,8)$. Previous studies have reported that thickness and volume of extraocular muscles (EOMs) and signal intensity ratio (SIR) of EOMs and orbital fat (OF) were associated with the treatment response of GC $(8,9)$. However, these structural and semi-quantitative parameters merely described the average value, while overlooked the heterogeneity within orbital tissues.

Texture analysis can provide objective and quantitative information about tissue heterogeneity and underlying pathologies (10). The distribution of gray-levels within an image can be reflected by a set of quantifiable metrics derived from texture analysis. To date, texture analysis has been mainly applied in the field of tumor imaging, for tumor subtype classification, lymph node metastases identification and cancer prognostic prediction $(11,12,13)$. However, to the best of our acknowledge, no study has applied MRI-based texture analysis in the evaluation of TAO patients.

Therefore, the purposes of our study were: (i) to determine whether the MRI texture features of EOM and OF could monitor the efficacy of GC therapy and (ii) to evaluate the value of the MRI texture features in predicting responsiveness to GC therapy in patients with active and moderate-to-severe TAO.

\section{Materials and methods}

\section{Patients}

This study was approved by the Ethics Committee of the First Affiliated Hospital of Nanjing Medical University and the informed consent was waived due to its retrospective nature. From November 2016 to December 2019, thirtyseven patients (mean age, $48.8 \pm 11.2$ years; male/ female ratio, 14/23) with clinically diagnosed active and moderate-to-severe TAO were enrolled. The inclusion criteria were as follows: (i) orbital MRI scans were available before and 6 months after the end of the GC treatment; (ii) image quality was adequate for subsequent analysis; (iii) disease duration was shorter than 18 months; (iv) no history of radiotherapy, surgical decompression or other immunosuppressive therapy; and (v) no other orbital diseases. All patients received a recommended intravenous GC therapy with cumulative $4.5 \mathrm{~g}$ glucocorticoid for 12 weeks ( $0.5 \mathrm{~g}$ weekly for 6 weeks, followed by $0.25 \mathrm{~g}$ weekly for 6 weeks) based on the European Group on Graves' Orbitopathy consensus (2).

\section{Clinical assessment}

Disease duration was determined as the interval between the onset of TAO-related ophthalmic symptoms and the date of the first MRI examination before treatment. Disease activity was evaluated based on the modified seven-point CAS. The eye with CAS $\geq 3$ was defined as active (6). Disease severity was assessed according to the NOSPECS scheme (14). All the above parameters were recorded for each eye.

All patients were assessed by the same endocrinologist and ophthalmologist before and 6 months after the end of the treatment. Results of assessments were compared to evaluate therapeutic outcomes. Then the eyes were divided into responders and unresponders. The responders were defined as (i) CAS dropped $\geq 2$ points and CAS <3; (ii) accompanied with at least one of the following improvements, without worsening of any others: decrease of proptosis $\geq 2 \mathrm{~mm}$; decrease of lid width $\geq 2 \mathrm{~mm}$; reduction by at least one class of the Gorman score; improvement of visual acuity $\geq 1$ Snellen line. The unresponders were defined as CAS dropping to less than 2 points or staying active (CAS $\geq 3 / 7$ ) $(4,15)$. Then, the $\triangle \mathrm{CAS} \%$ was calculated by the formula: $\Delta \mathrm{CAS} \%=\left(\mathrm{CAS}_{\text {pre }}-\mathrm{CAS}_{\text {post }}\right) / \mathrm{CAS}_{\text {pre }} \times 100 \%$, in order to reflect the rate of change of CAS after treatment.
This work is licensed under a Creative Commons Attribution-NonCommercial 4.0 International License. ded from Bioscientifica.com at 04/26/2023 01:07:56PM 


\section{Image acquisition}

MRI scans were carried out on a 3.0-T MRI system (Magnetom Skyra; Siemens Healthcare, Erlangen, Germany) with a 20-channel head coil. MRI protocols consisted of the following sequences: axial T1- weighted image (repetition time/echo time, 635/6.7 ms), and axial, coronal, and sagittal T2-weighted images with fat suppression (FS) (repetition time/echo time, 4000/75-117 ms). The other parameters include: (i) axial T2-weighted image with FS and T1-weighted image: field of view, $200 \times 200 \mathrm{~mm}^{2}$; matrix, $320 \times 320$; slice thickness, $3.0 \mathrm{~mm}$; (ii) Coronal T2-weighted image with FS: field of view, $180 \times 180 \mathrm{~mm}^{2}$; matrix, $224 \times 320$; slice thickness, $3.5 \mathrm{~mm}$; (iii) Sagittal T2-weighted image with FS: field of view, $180 \times 180 \mathrm{~mm}^{2}$; matrix, $346 \times 384$; slice thickness, $3.5 \mathrm{~mm}$.

\section{Imaging analysis}

Two radiologists, with 10 and 3 years of experience in head and neck imaging, respectively, carried out image analysis independently. The observers were blinded to study design and clinical information. The measurement results of two observers were used to assess the inter-observer agreement. One monthlater, the first observer repeated themeasurement for assessing the intra-observer reproducibility. Imaging data were analyzed in each unit of the eye.

Placement of region of interests (ROIs) was performed on ITK-SNAP software (www.itksnap.org). Based on coronal T2-weighted image with FS, slices behind the eyeball, which represent the largest area of muscle bellies and intraorbital fat were chosen (Fig. 1A and B). Polygonal ROIs were drawn manually on EOMs and OF, respectively. After ROIs were placed, following quantitative texture parameters of EOMs and $\mathrm{OF}$, including the entropy, uniformity, skewness and kurtosis, were obtained automatically by using AK software (Artificial Intelligence Kit, GE Healthcare) (16).

In addition, the thickness of EOMs and OF was obtained by the following method: (1) EOM - vertical diameters of superior and inferior EOMs and horizontal diameters of the medial and lateral EOMs were measured on coronal T2-weighted image with FS. The maximum values of four diameters were recorded (Fig. 1C). (2) OF - the maximum distance between the medial wall of the eyeball and the medial wall of the orbit on axial T1-weighted image was measured (Fig. 1D). Then, the rate of change for every imaging parameter before and after treatment was calculated by adopting the following formula: $\Delta \mathrm{MRI} \%=\left(\mathrm{MRI}_{\text {pre }}-\mathrm{MRI}_{\text {post }}\right) / \mathrm{MRI}_{\text {pre }} \times 100 \%$.

\section{Statistical analysis}

All numeric data were expressed as mean \pm S.D. The normality distribution was assessed using KolmogorovSmirnov test. Paired $t$ test was used to compare the parameters before and after treatment. Chi-squared test was used to analyze the difference of categorical data between groups. Spearman's rank correlation coefficient was used to evaluate the correlation between $\Delta C A S \%$ and the rate of changes of imaging parameters. Independent-sample t-test and Mann-Whitney U test were applied to compare the pre-treatment clinical and imaging parameters between responders and unresponders. All the variables with a $P$ value less than 0.01 during univariate analysis were included in the multivariate logistic regression model to determine the independent predictors of treatment response. Finally, receiver operating characteristic (ROC) curves analyses were conducted to determine the
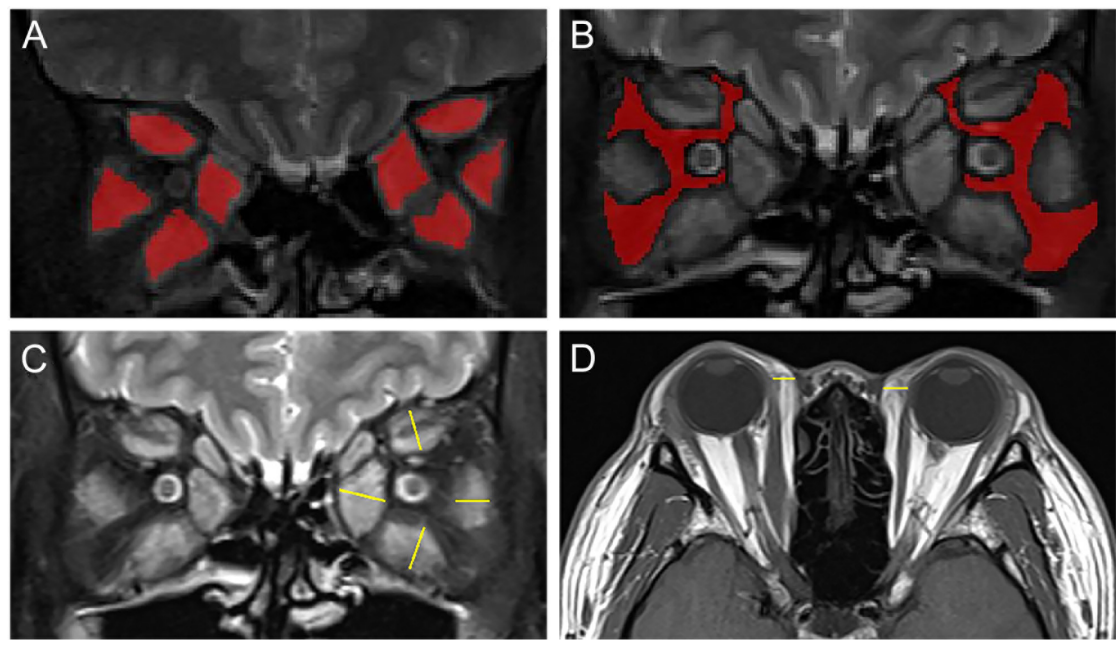

\section{Figure 1}

Schematic diagram for the measurement of orbital MRI-based quantitative parameters. (A and B) showed the region of interest placement (in red) of EOM and OF on coronal T2-weighted images with FS using ITK-SNAP software. (C and D) showed the quantitative measurements regarding the thickness of EOM and OF on coronal

T2-weighted image with FS and axial T1-weighted image, respectively. EOM, extraocular muscle; OF, orbital fat; FS, fat suppression. https://ec.bioscientifica.com https://doi.org/10.1530/EC-21-0162 (c) 2021 The authors Published by Bioscientifica Ltd

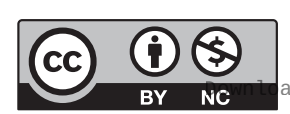

This work is licensed under a Creative Commons Attribution-NonCommercial 4.0 International License. ded from Bioscientifica.com at 04/26/2023 01:07:56PM 
diagnostic performance of identified variables and their combinations for predicting the curative effect.

Intraclass correlation coefficients (ICC) with 95\% CIs were used to evaluate the inter- and intra-observer reproducibility of the measurements, and a two-way method with random rater assumption was applied. ICC was interpreted as follows: $<0.40$, poor; $0.40-0.60$, moderate; $0.61-0.80$, good; and $\geq 0.81$, excellent. The significance threshold for differences on imaging parameters was defined as two-sided $P<0.01(0.05 / 5)$ for Bonferroni multiple comparisons correction. All statistical analyses were conducted by using the SPSS software (v. 23.0; IBM) and MedCalc (v. 19.0; Ostend, Belgium).

\section{Results}

\section{Clinical data between responders and unresponders}

Detailed demographic and clinical information of our study cohort are listed in Table 1 . The average disease duration of responders was significantly shorter than that of the unresponders $(4.2 \pm 2.6$ vs $8.6 \pm 5.5$ months, $P=0.003)$. No significant differences were found in other clinical parameters (age, sex distribution, smoking history, pre-treatment thyroid function, serum TRAb, CAS, and diplopia presence) between responders and unresponders (all $P>0.05$ ).

\section{Variables for monitoring the response to GC therapy}

\section{Differences of imaging parameters before and after treatment}

Good to excellent inter-observer and excellent intraobserver reproducibility were achieved during the measurements of all parameters (ICCs: 0.706-0.975, 0.813-0.980, respectively). Responsive TAO patients showed significantly decreased entropy and increased uniformity at $\mathrm{EOM}$ and $\mathrm{OF}$, and decreased maximum thickness of EOM after GC therapy (all $P<0.01$ ) (Tables 2 and 3, Fig. 2). No significant differences were found in the unresponders regarding all the imaging parameters (all $P>0.01$ ).

\section{Correlations between changes of imaging parameters and CAS before and after treatment}

The $\Delta$ entropy $\%$ of EOM and OF were positively correlated with $\Delta$ CAS\% $(r=0.379,0.263 ; P=0.001,0.024$, respectively $)$, and $\Delta$ uniformity $\%$ of those negatively correlated with $\Delta$ CAS\% $(\mathrm{r}=-0.386,-0.256 ; P=0.001,0.028$; respectively) (Fig. 3). Besides, $\Delta$ maximum thickness\% of EOM showed significantly positive correlation with $\Delta$ CAS\% $(r=0.347$, $P=0.002)$, while that of OF not $(P>0.05)$.

\section{Variables for predicting the response to GC therapy}

\section{Differences of pre-treatment imaging parameters between responders and unresponders}

Responders showed significantly lower entropy and higher uniformity at EOM compared with unresponders before treatment (all $P<0.01$ ) (Table 2, Fig. 2). However, there was no significant difference in pre-treatment parameters at $\mathrm{OF}$ between two groups (all $P>0.01$ ) (Table 3 ).

\section{Multivariate analysis and ROC curves analyses}

Multivariate logistic regression analysis showed that both entropy and uniformity of EOM and disease duration were independent predictors for responsive TAOs $(P=0.010$, $0.029,0.003$, respectively). Combined indicators including

Table 1 Detailed clinical and demographic information of our study population. The numeric data are reported as the mean \pm s.D. (range).

\begin{tabular}{lcc}
\hline Variables & Responders $(n=23)$ \\
\cline { 1 - 2 } Age (years) & $49.5 \pm 10.2(32-65)$ \\
Sex (F/M) & $15 / 8$ \\
Smoking history & $6 / 17$ \\
Disease duration (months) & $4.2 \pm 2.6(1-12)$ \\
CAS & $4.0 \pm 0.8(3-6)$ \\
FT3 (pmol/L) & $5.86 \pm 2.76(3.67-14.10)$ \\
FT4 (pmol/L) & $19.50 \pm 8.32(10.34-42.93)$ \\
TSH (mIU/L) & $1.540 \pm 2.293(0.005-9.700)$ \\
TRAb (IU/L) & $8.96 \pm 10.19(0.37-40.00)$ \\
Diplopia presence & $12 / 11$ \\
\hline
\end{tabular}

\begin{tabular}{|c|c|}
\hline Unresponders $(n=14)$ & $\boldsymbol{P}$ \\
\hline $47.6 \pm 13.1(25-67)$ & 0.637 \\
\hline $8 / 6$ & 0.732 \\
\hline $5 / 9$ & 0.713 \\
\hline $8.6 \pm 5.5(4-17)$ & $0.003^{a}$ \\
\hline $3.9 \pm 0.8(3-5)$ & 0.840 \\
\hline $6.01 \pm 2.95(3.66-14.80)$ & $>0.999$ \\
\hline $21.06 \pm 10.04(11.12-44.30)$ & 0.754 \\
\hline $1.130 \pm 1.372(0.005-3.290)$ & 0.975 \\
\hline $9.22 \pm 10.20(0.10-40.00)$ & 0.695 \\
\hline $11 / 3$ & 0.166 \\
\hline
\end{tabular}

${ }^{a}$ Statistical significance is indicated by $P$ values $<0.05$.

CAS, clinical activity score; F, female; FT3, free triiodothyronine; FT4, free thyroxine; M, male; TAO, thyroid-associated ophthalmopathy; TRAb, thyroidstimulating hormone receptor antibody; TSH, thyroid-stimulating hormone. 


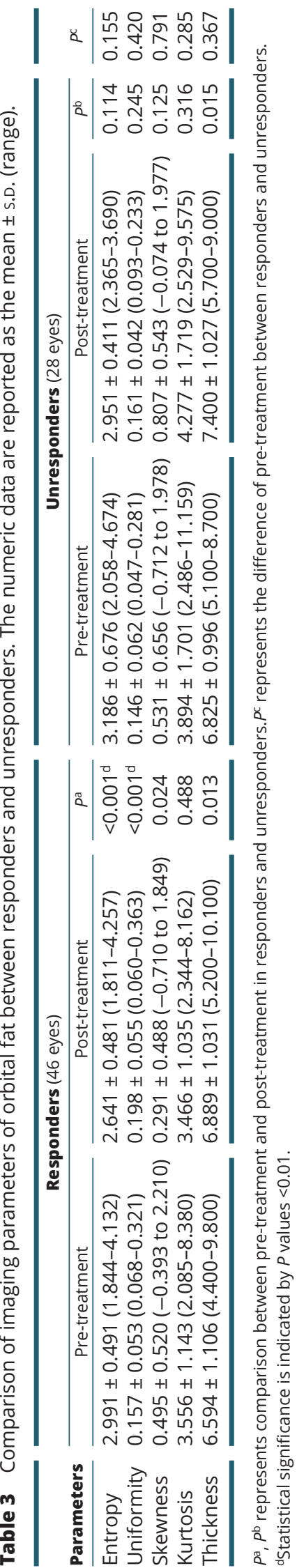


A.EOM
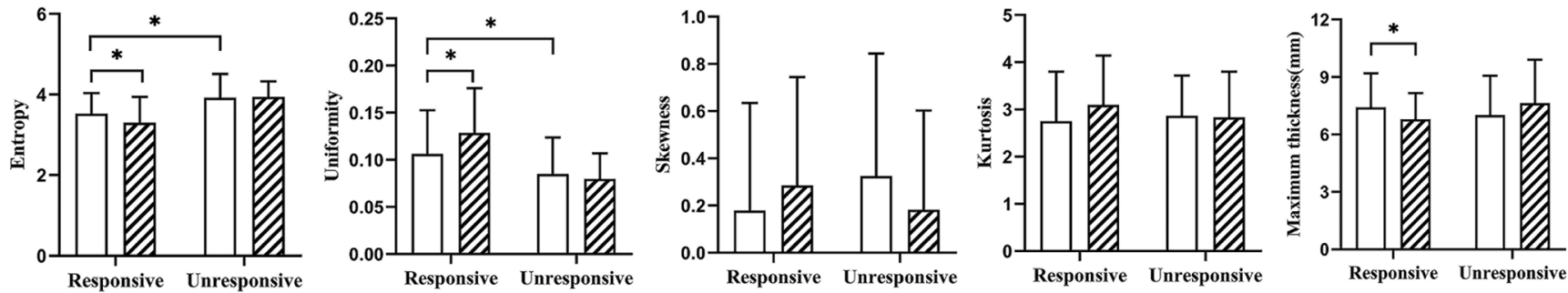

B.OF

$\square$ Pre-treatment ש Post-treatment
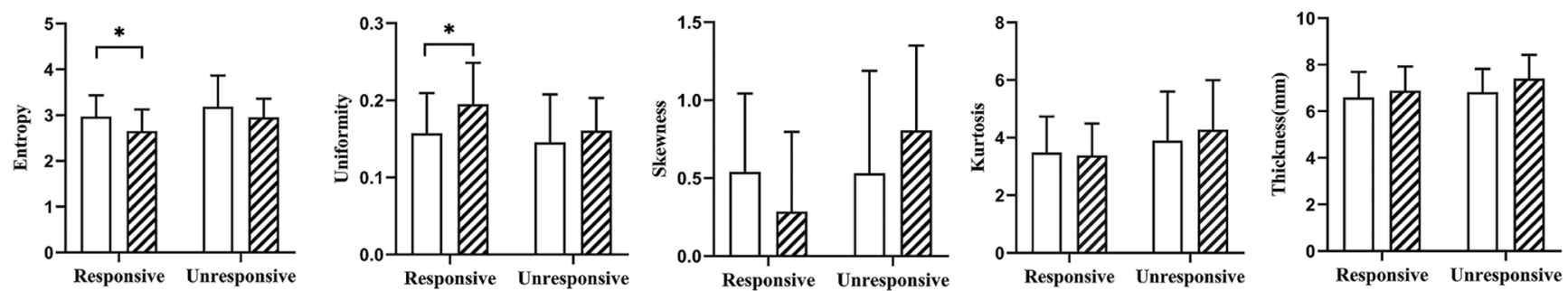

Figure 2

Comparisons of orbital MRI-based parameters before and after treatment. Asterisk indicated significant differences $(* P<0.01)$. EOM, extraocular muscle; OF, orbital fat.

\section{A.EOM}

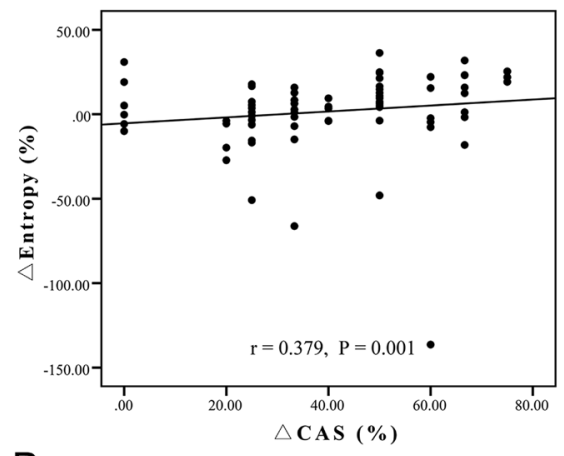

B.OF

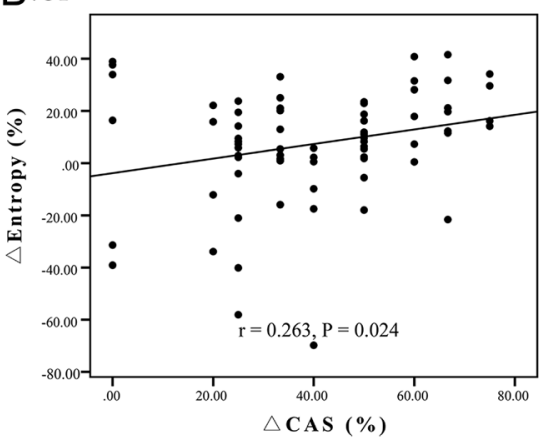

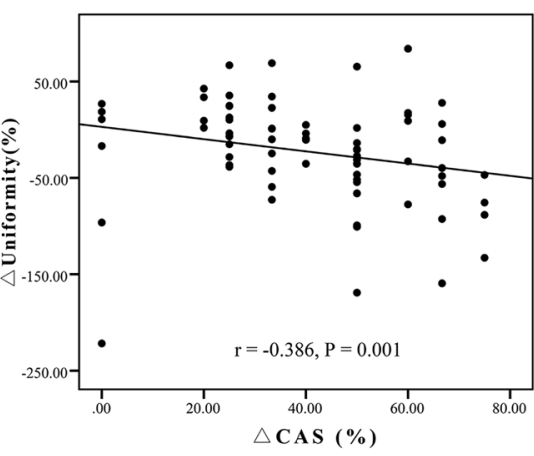
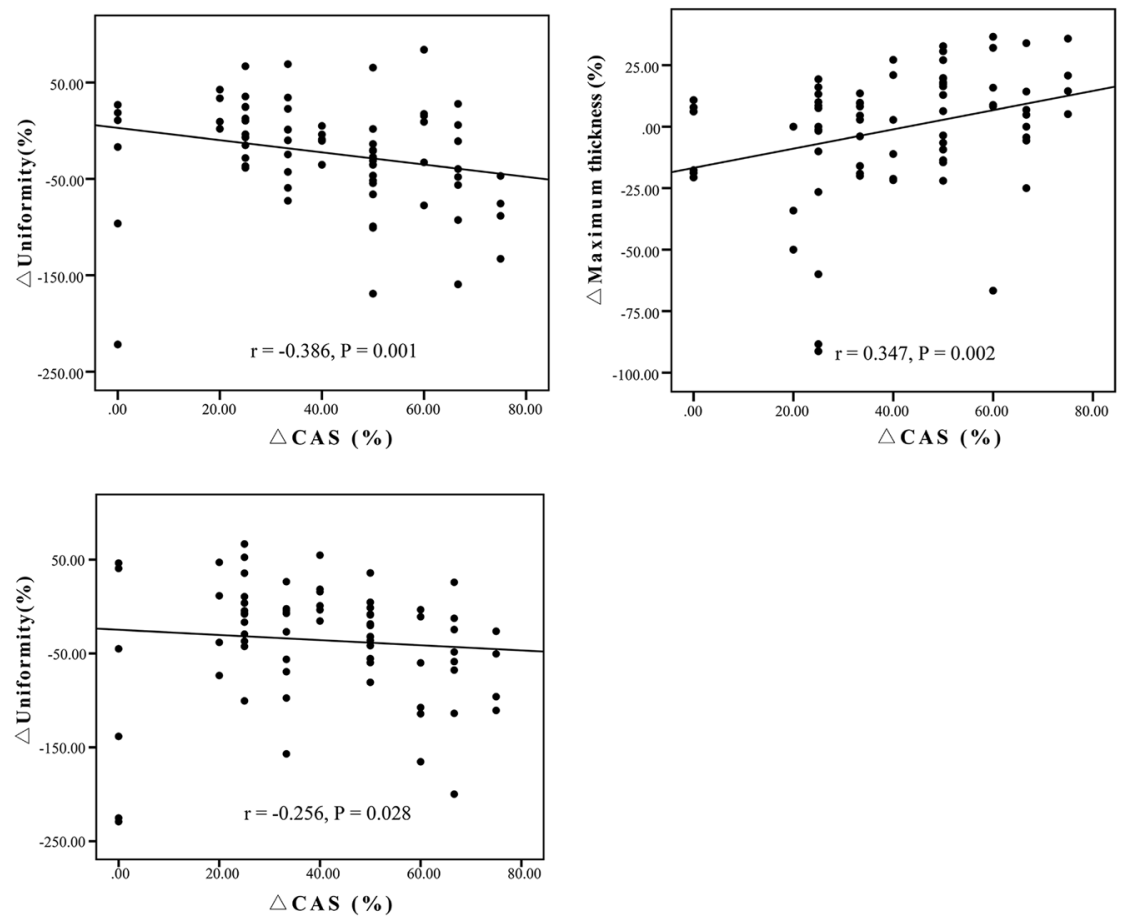

Figure 3

Correlation analyses between changes of imaging parameters and that of CAS in TAO patients. EOM, extraocular muscle; OF, orbital fat; CAS, clinical activity score. $\begin{array}{lr}\text { https://ec.bioscientifica.com } & \text { (c) } 2021 \text { The authors } \\ \text { https://doi.org/10.1530/EC-21-0162 } & \text { Published by Bioscientifica Ltd }\end{array}$
This work is licensed under a Creative Commons Attribution-NonCommercial 4.0 International License. ded from Bioscientifica.com at 04/26/2023 01:07:56PM 
Table 4 Predictive performance of identified variables and their combinations for responsive TAOs. The unit of DD is month.

\begin{tabular}{l} 
Model \\
\hline EOM - Entropy \\
EOM - Uniformity \\
DD \\
DD + Entropy + Uniformity
\end{tabular}

\begin{tabular}{c}
\hline AUC $(95 \% \mathrm{Cl})$ \\
\hline $0.705(0.588-0.805)$ \\
$0.684(0.566-0.787)$ \\
$0.795(0.685-0.880)$ \\
$0.802(0.693-0.886)$ \\
\hline
\end{tabular}

\begin{tabular}{l} 
Cut-off \\
\hline$\leq 3.855$ \\
$\geq 0.081$ \\
$\leq 3.0$ \\
$\quad-$
\end{tabular}

\begin{tabular}{c}
\hline Sensitivity, \% $(95 \% \mathrm{CI})$ \\
\hline $80.4(66.1-90.6)$ \\
$80.4(66.1-90.6)$ \\
$52.2(36.9-67.1)$ \\
$82.6(68.6-92.2)$ \\
\hline
\end{tabular}

\begin{tabular}{l} 
Specificity, $\%(95 \% \mathrm{Cl})$ \\
\hline $60.7(40.6-78.5)$ \\
$64.3(44.1-81.4)$ \\
$100(87.7-100.0)$ \\
$67.9(47.6-84.1)$
\end{tabular}

AUC, area under curve; DD, disease duration; EOM, extraocular muscle; TAO, thyroid-associated ophthalmopathy.

entropy and uniformity of EOM and disease duration using multivariate analysis gained an area under curve of 0.802 , a sensitivity of $82.6 \%$, and a specificity of $67.9 \%$ for predicting responsive TAOs. Singly using disease duration $\leq 3.0$ months, optimal predictive specificity (100\%) was obtained. Detailed ROC curve analyses results were displayed in Table 4 and Fig. 4.

\section{Discussion}

Present study had two main findings. First, entropy and uniformity of EOM and OF, as well as maximum thickness of EOM, differed significantly before and after treatment in the responders. Changes of entropy and uniformity of EOM and OF, as well as that of maximum thickness at EOM, correlated significantly with changes of CAS. Second, pre-treatment entropy and uniformity of EOM differed significantly between responders and unresponders.

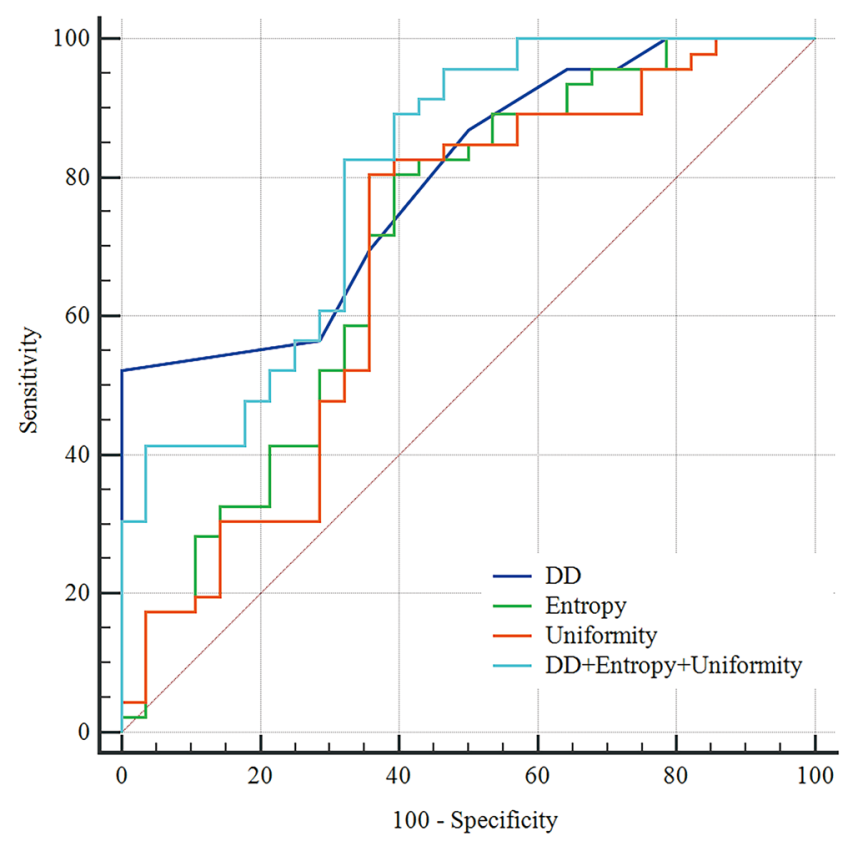

Figure 4

Receiver operating characteristic curves of identified variables and their combination for predicting responsive TAOs. DD, disease duration.
Pre-treatment entropy and uniformity of EOM and disease duration were independent predictors for responsive TAOs. Combination of three indicators achieved optimal efficacy for predicting responsive TAOs. Our study indicated that MRI-based texture parameters of EOM and OF, especially those of EOM, could serve as promising markers for concurrently monitoring and predicting the response of GC therapy in TAO patients, and help clinicians to make individualized treatment plans.

Previously, Tomoaki et al. reported that SIR of EOM in TAO patients decreased significantly after GC therapy, while patients with deteriorated symptoms would have one or more EOMs kept high SIR after treatment $(8,17)$. They indicated that SIR of EOMs could be useful for evaluating the efficacy of treatment in TAO patients. However, the semi-quantitative and averaged value of signal intensity used in their study was easily influenced by the subjectivity of ROI selection method. More importantly, it ignored the heterogeneity within orbital tissues. In the present study, we performed MRI-based texture analysis so as to excavate more information associated with tissue heterogeneity. Our results indicated that responders had increased uniformity and decreased entropy of EOM and OF after treatment. Previously, uniformity and entropy have been reported to be associated with the degree of tissue homogeneity and heterogeneity, respectively, in various organs and diseases $(18,19,20,21)$. Meanwhile, growing evidence showed that GC therapy could reduce inflammation and increase the uniformity of orbital tissues in the active TAO patients $(22,23)$. Thus, our results are in accord with previous suggestions, and indicated that signal intensity of both EOM and OF tend to be more uniform after treatment in the responders.

Moreover, significant positive correlation between $\Delta$ entropy $\%$ and $\triangle \mathrm{CAS} \%$, while a negative correlation between $\Delta$ uniformity $\%$ and $\triangle \mathrm{CAS} \%$ were found at both EOM and OF. In clinical practice, CAS is a simple and widely used method for assessing the treatment response in TAO patients. However, its main disadvantage is subjective and partially anterior-orbital evaluation, which may make it difficult for clinicians to determine the therapeutic 
effect accurately. Our correlation results verified the robustness of orbital MRI-based texture for monitoring the treatment response in TAO patients. Our study may lay the foundation of adding MRI-derived texture parameters into the monitoring system besides CAS in the future.

Another important finding in our study was that, responders showed lower entropy and higher uniformity of EOM before treatment compared with unresponders. Previously, one study has observed that the T2-signal intensity in enlarged EOM was not always elevated homogenously, and it was supposed to indicate the coexistence of different pathological manifestations in EOM. However, they could not provide objective and quantifiable evidence (8). Recently, two studies reported that responsive TAO patients had a lower standard deviation of signal intensity at EOM on T2-weighted image, which reflected that the EOM signal was more uniform in responsive patients than unresponsive mimics $(24,25)$. TAO usually involves a transition from an active phase to a stationary phase, and the progression of fibrosis is often accompanied by an increase in the heterogeneity of the affected regions $(8,26,27,28)$. Therefore, we deduced that our current finding might also indicate the increased portion of fibrosis combined with increased heterogeneity within orbital tissues in unresponsive TAOs, after considering the natural pathology of the disease and the longer duration of unresponsive cases.

Furthermore, the entropy and uniformity of EOM, together with disease duration, were identified as independent predictors for treatment response in this study. Notably, although extremely specific (100\%), the disease duration was not sensitive enough (52.2\%) in predicting the therapeutic response. Combination usage of all three independent predictors could achieve an optimal efficiency with improved sensitivity (82.6\%). Clinically, it is crucial to distinguish between responders and unresponders before GC therapy. The determined responsive patients would accept GC therapy, while other therapies, such as other kinds of immunosuppressive therapies and even surgery, would be suggested to unresponsive patients. Our results indicated that MRI-based texture analysis could be used as an effective and objective method for early prediction and monitoring of treatment response in TAO patients, which could help clinicians to develop individualized therapeutic strategy.

There were several limitations of this study. First, this is a single-center retrospective design with a relatively small sample size. Thus, the power and generalizability of our result need to be verified. Second, we only include specific populations with disease duration less than 18 months, which may result in potential selection bias. Third, histological assessments of orbital tissues in TAO patients were not available due to the retrospective nature. The corresponding relationship between imaging parameters and pathological changes cannot be accurately verified. Further investigations combining multi-parameter MRI and CAS, or conducting a larger and multi-center prospective collection, or enrolling different TAO populations would be more comprehensive and significant, in order to validate our results and provide conclusive evidence for this issue.

In conclusion, we employed orbital MRI-based texture analysis to monitor and predict the GC therapeutic response in TAO patients. Our results suggested that orbital MRI-based texture parameters, especially that of EOM, could be a useful and clinically applicable method to guide the treatment plan and advance precision medicine for TAO patients.

\section{Declaration of interest}

The authors declare that there is no conflict of interest that could be perceived as prejudicing the impartiality of the research reported.

\section{Funding}

This work was supported by National Natural Science Foundation of China (NSFC) (No. 81801659) to Hao Hu and 'Thyroid Research Program of Young and Middle-aged Physicians' from China Health Promotion Foundation to Huan-Huan Chen.

\section{References}

1 Bahn RS. Graves' ophthalmopathy. New England Journal of Medicine 2010362 726-738. (https://doi.org/10.1056/NEJMra0905750)

2 Bartalena L, Baldeschi L, Boboridis K, Eckstein A, Kahaly GJ, Marcocci C, Perros P, Salvi M, Wiersinga WM \& European Group on Graves. The 2016 European Thyroid Association/European Group on Graves' orbitopathy guidelines for the management of Graves' orbitopathy. European Thyroid Journal 20165 9-26. (https://doi. org/10.1159/000443828)

3 Zang S, Ponto KA \& Kahaly GJ. Clinical review: intravenous glucocorticoids for Graves' orbitopathy: efficacy and morbidity. Journal of Clinical Endocrinology and Metabolism 201196 320-332. (https://doi. org/10.1210/jc.2010-1962)

4 Vannucchi G, Covelli D, Campi I, Origo D, Curro N, Cirello V, Dazzi D, Beck-Peccoz P \& Salvi M. The therapeutic outcome to intravenous steroid therapy for active Graves' orbitopathy is influenced by the time of response but not polymorphisms of the glucocorticoid receptor. European Journal of Endocrinology 2014170 55-61. (https://doi. org/10.1530/EJE-13-0611)

5 Miskiewicz P, Kryczka A, Ambroziak U, Rutkowska B, Glowczynska R, Opolski G, Kahaly G \& Bednarczuk T. Is high dose intravenous methylprednisolone pulse therapy in patients with Graves'
This work is licensed under a Creative Commons Attribution-NonCommercial 4.0 International License. ed from Bioscientifica.com at 04/26/2023 01:07:56PM via free access 
orbitopathy safe? Endokrynologia Polska 201465 402-413. (https://doi. org/10.5603/EP.2014.0056)

6 Mourits MP, Prummel MF, Wiersinga WM \& Koornneef L. Clinical activity score as a guide in the management of patients with Graves' ophthalmopathy. Clinical Endocrinology 199747 9-14. (https://doi. org/10.1046/j.1365-2265.1997.2331047.x)

7 Zhou M, Shen L, Jiao Q, Ye L, Zhou Y, Zhu W, Wang W \& Wang S. Role of magnetic resonance imaging in the assessment of active thyroidassociated ophthalmopathy patients with long disease duration. Endocrine Practice 201925 1268-1278. (https://doi.org/10.4158/EP-20190133)

8 Yokoyama N, Nagataki S, Uetani M, Ashizawa K \& Eguchi K. Role of magnetic resonance imaging in the assessment of disease activity in thyroid-associated ophthalmopathy. Thyroid 200212 223-227. (https://doi.org/10.1089/105072502753600179)

9 Higashiyama T, Nishida Y \& Ohji M. Changes of orbital tissue volumes and proptosis in patients with thyroid extraocular muscle swelling after methylprednisolone pulse therapy. Japanese Journal of Ophthalmology 201559 430-435. (https://doi.org/10.1007/s10384-015-0410-4)

10 De Cecco CN, Ganeshan B, Ciolina M, Rengo M, Meinel FG, Musio D, De Felice F, Raffetto N, Tombolini V \& Laghi A. Texture analysis as imaging biomarker of tumoral response to neoadjuvant chemoradiotherapy in rectal cancer patients studied with 3-T magnetic resonance. Investigative Radiology 201550 239-245. (https:// doi.org/10.1097/RLI.0000000000000116)

11 Su GY, Xu XQ, Zhou Y, Zhang H, Si Y, Shen MP \& Wu FY. Texture analysis of dual-phase contrast-enhanced CT in the diagnosis of cervical lymph node metastasis in patients with papillary thyroid cancer. Acta Radiologica 2020 284185120946711. (https://doi. org/10.1177/0284185120946711)

12 Aker M, Ganeshan B, Afaq A, Wan S, Groves AM \& Arulampalam T. Magnetic resonance texture analysis in identifying complete pathological response to neoadjuvant treatment in locally advanced rectal cancer. Diseases of the Colon and Rectum 201962 163-170. (https://doi.org/10.1097/DCR.0000000000001224)

13 Fritz B, Muller DA, Sutter R, Wurnig MC, Wagner MW, Pfirrmann CWA $\&$ Fischer MA. Magnetic resonance imaging-based grading of cartilaginous bone tumors: added value of quantitative texture analysis. Investigative Radiology 201853 663-672. (https://doi. org/10.1097/RLI.0000000000000486)

14 Dolman PJ. Evaluating Graves' orbitopathy. Best Practice and Research: Clinical Endocrinology and Metabolism 201226 229-248. (https://doi. org/10.1016/j.beem.2011.11.007)

15 Hu S, Wang Y, He M, Zhang M, Ding X \& Shi B. Factors associated with the efficacy of intravenous methylprednisolone in moderate-tosevere and active thyroid-associated ophthalmopathy: a single-centre retrospective study. Clinical Endocrinology 201990 175-183. (https:// doi.org/10.1111/cen.13855)

16 Hocquelet A, Denis de Senneville B, Frulio N, Salut C, Bouzgarrou M, Papadopoulos P \& Trillaud H. Magnetic resonance texture parameters are associated with ablation efficiency in MR-guided high-intensity focussed ultrasound treatment of uterine fibroids. International Journal of Hyperthermia 201733 142-149. (https://doi.org/10.1080/02656736.2016. 1241432)

17 Higashiyama T, Nishida Y, Morino K, Ugi S, Nishio Y, Maegawa H \& Ohji M. Use of MRI signal intensity of extraocular muscles to evaluate methylprednisolone pulse therapy in thyroid-associated ophthalmopathy. Japanese Journal of Ophthalmology 201559 124-130. (https://doi.org/10.1007/s10384-014-0365-x)

18 Giganti F, Antunes S, Salerno A, Ambrosi A, Marra P, Nicoletti R, Orsenigo E, Chiari D, Albarello L, Staudacher C et al. Gastric cancer: texture analysis from multidetector computed tomography as a potential preoperative prognostic biomarker. European Radiology 2017 27 1831-1839. (https://doi.org/10.1007/s00330-016-4540-y)

19 Lakhman Y, Veeraraghavan H, Chaim J, Feier D, Goldman DA, Moskowitz CS, Nougaret S, Sosa RE, Vargas HA, Soslow RA et al. Differentiation of uterine leiomyosarcoma from atypical leiomyoma: diagnostic accuracy of qualitative MR imaging features and feasibility of texture analysis. European Radiology 201727 2903-2915. (https:// doi.org/10.1007/s00330-016-4623-9)

20 Kim JH, Ko ES, Lim Y, Lee KS, Han BK, Ko EY, Hahn SY \& Nam SJ. Breast cancer heterogeneity: MR imaging texture analysis and survival outcomes. Radiology 2017282 665-675. (https://doi.org/10.1148/ radiol.2016160261)

21 Deng Y, Soule E, Samuel A, Shah S, Cui E, Asare-Sawiri M, Sundaram C, Lall C \& Sandrasegaran K. CT texture analysis in the differentiation of major renal cell carcinoma subtypes and correlation with Fuhrman grade. European Radiology 201929 6922-6929. (https://doi. org/10.1007/s00330-019-06260-2)

22 Liu P, Chen L, Wang QX, Luo B, Su HH, Yuan G, Jiang GH \& Zhang J. Histogram analysis of T2 mapping for detecting early involvement of extraocular muscles in patients with thyroid-associated ophthalmopathy. Scientific Reports 202010 19445. (https://doi. org/10.1038/s41598-020-76341-6)

23 Taylor PN, Zhang L, Lee RWJ, Muller I, Ezra DG, Dayan CM, Kahaly GJ \& Ludgate M. New insights into the pathogenesis and nonsurgical management of Graves orbitopathy. Nature Reviews: Endocrinology 2020 16 104-116. (https://doi.org/10.1038/s41574-019-0305-4)

24 Ito M, Takahashi Y, Katsuda E, Oshima Y, Takeuchi A, Mori T, Abe S, Mori Y, Kakizaki H \& Suzuki K. Predictive factors of prognosis after radiation and steroid pulse therapy in thyroid eye disease. Scientific Reports 20199 2027. (https://doi.org/10.1038/s41598-01938640-5)

$25 \mathrm{Hu}$ H, Xu XQ, Chen L, Chen W, Wu Q, Chen HH, Zhu H, Shi HB $\&$ Wu FY. Predicting the response to glucocorticoid therapy in thyroid-associated ophthalmopathy: mobilizing structural MRI-based quantitative measurements of orbital tissues. Endocrine $2020 \mathbf{7 0}$ 372-379. (https://doi.org/10.1007/s12020-020-02367-5)

26 Potgieser PW, Wiersinga WM, Regensburg NI \& Mourits MP. Some studies on the natural history of Graves' orbitopathy: increase in orbital fat is a rather late phenomenon. European Journal of Endocrinology 2015173 149-153. (https://doi.org/10.1530/EJE-141140)

$27 \mathrm{Hu}$ H, Chen HH, Chen W, Wu Q, Chen L, Zhu H, Xu XQ, Shi HB \& Wu FY. T2 mapping histogram at extraocular muscles for predicting the response to glucocorticoid therapy in patients with thyroidassociated ophthalmopathy. Clinical Radiology 202176 159.e1-159.e8. (https://doi.org/10.1016/j.crad.2020.09.005)

28 Bartalena L, Piantanida E, Gallo D, Lai A \& Tanda ML. Epidemiology, natural history, risk factors, and prevention of Graves orbitopathy. Frontiers in Endocrinology 202011 615993. (https://doi.org/10.3389/ fendo.2020.615993)

Received in final form 21 May 2021

Accepted 2 June 2021

Accepted Manuscript published online 2 June 2021 https://ec.bioscientifica.com https://doi.org/10.1530/EC-21-0162 (c) 2021 The authors Published by Bioscientifica Ltd

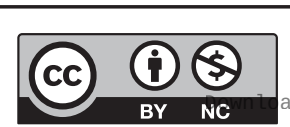

This work is licensed under a Creative Commons Attribution-NonCommercial 4.0 International License. ded from Bioscientifica.com at 04/26/2023 01:07:56PM 